\title{
Changes in Hind Paw Epidermal Thickness, Peripheral Nerve Distribution and Mechanical Sensitivity After Immobilization in Rats
}

\author{
J. NAKANO ${ }^{1}$, Y. SEKINO ${ }^{2}$, Y. HAMAUE ${ }^{2}$, J. SAKAMOTO ${ }^{3}$, T. YOSHIMURA ${ }^{2}$, \\ T. ORIGUCHI ${ }^{2}$, M. OKITA ${ }^{2}$
}

${ }^{1}$ Unit of Physical Therapy and Occupational Therapy Sciences, Nagasaki University Graduate School of Biochemical Sciences, Nagasaki-shi, Japan, ${ }^{2}$ Department of Locomotive Rehabilitation Science, Unit of Rehabilitation Sciences, Nagasaki University Graduate School of Biomedical Sciences, Nagasaki-shi, Japan, ${ }^{3}$ Department of Rehabilitation, Nagasaki University Hospital, Nagasaki-shi, Japan

Received March 22, 2012

Accepted July 23, 2012

On-line October 25, 2012

\begin{abstract}
Summary
This study was designed to investigate histological changes in skin tissue accompanying immobilization-induced hypersensitivity. Changes in mechanical sensitivity, epidermal thickness, and peripheral nerve profiles in the upper dermis were examined in glabrous skin of rat hind paw after 1, 2, and 4 weeks of ankle joint immobilization by plaster casts. Induction of mechanical hypersensitivity was confirmed after 2 and 4 weeks of joint immobilization. Epidermal thinning and increase in peripheral nerve profiles were observed in skin tissues in immobilized rats. The time course of epidermal thinning and increase in peripheral nerve profiles were similar closely to that of hypersensitivity, with significant differences between the immobilized and control rats after 2 weeks of immobilization, which became even more remarkable at 4 weeks of immobilization. These findings suggest that joint immobilization by cast induces epidermal thinning and increases peripheral nerve profiles in the upper dermis and that these changes might be partly responsible for immobilization-induced hypersensitivity.
\end{abstract}

\section{Key words}

Immobilization • Hypersensitivity • Skin • Epidermal thickness • Peripheral nerve

\section{Corresponding author}

J. Nakano, Unit of Physical Therapy and Occupational Therapy Sciences, Nagasaki University Graduate School of Biochemical
Sciences, 1-7-1 Sakamoto, Nagasaki-shi, 852-8520, Japan. Tel. and Fax: +81-95-819-7919. E-mail: nakano-j@nagasaki-u.ac.jp

Joint immobilization is commonly used for the treatment of joint injuries, such as periarticular fractures and ligament injuries, but it also causes various degenerative or atrophic changes in intact organs and tissues, including muscular disuse atrophy, joint contractures, and articular cartilage degeneration in particular. In addition, immobilization is often associated with the induction of hypersensitivity. It has been reported that joint immobilization by cast induces hypersensitivity in humans (Terkelsen et al. 2008) and rats (Ushida and Willis 2001, Guo et al. 2004). In terms of the mechanism, several observations suggest that central nervous system changes may be responsible (Ushida and Willis 2001, Guo et al. 2004). As the precise mechanism underlying immobilization-induced hypersensitivity remains unclear, the possibility that changes in peripheral tissues including the skin may be involved should not be excluded.

Cutaneous atrophic changes are often observed after immobilization by casts in patients with fracture. Few experimental studies have dealt with this change; thus, the cutaneous histological changes caused by immobilization and their influence on hypersensitivity 
A

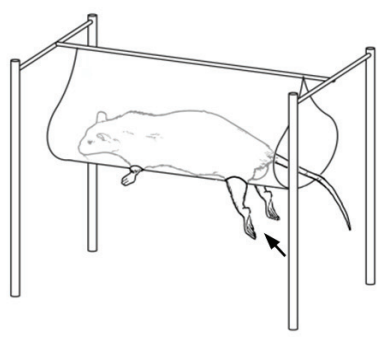

B

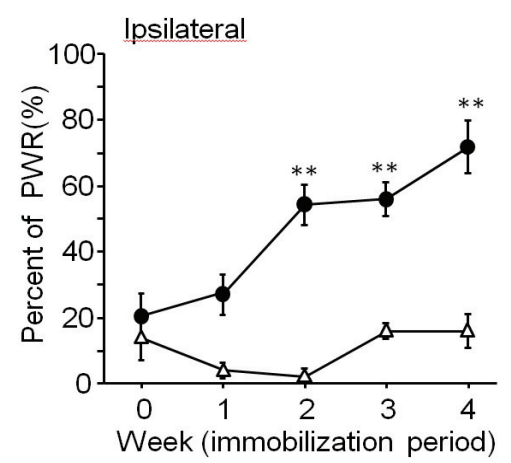

C

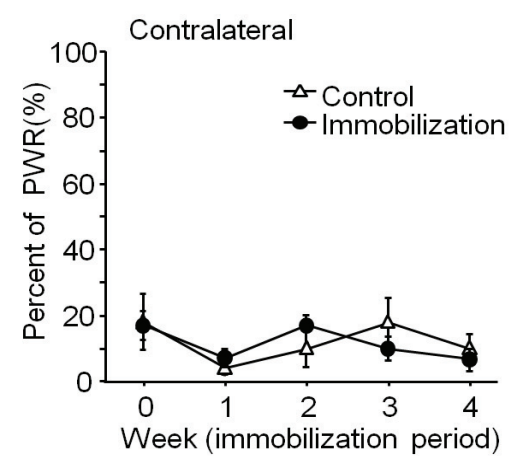

Fig. 1. The rats were placed individually in the restrainer so that the rear legs were under no loading (arrow), and the movement of legs was observed with certainty (A). Behavioral test for mechanical hypersensitivity in the joint-immobilized and control rats at the $15 \mathrm{~g}$ threshold. In the ipsilateral (right) paw of the immobilization group, the percent of paw withdrawal response (PWR) increased significantly after 2 weeks of immobilization compared with the control group and continued increasing until 4 weeks after immobilization (B). No increase in the contralateral (left) paw in both groups was observed $(\mathbf{C})(* * p<0.001)$.

remain unclear. More studies are necessary to investigate the mechanism underlying immobilization-induced hypersensitivity, and more specifically, studies regarding the time course of cutaneous tissue changes are particularly lacking. To address these issues, changes in the mechanical sensitivity of the skin, epidermal thickness, and peripheral nerve distribution in the upper dermis were examined in the present study after 1,2 , and 4 weeks of ankle joint immobilization in rats.

This study was approved by the Ethics Review Committee for Animal Experimentation of Nagasaki University. Forty-five 8-week-old male Wistar rats obtained from Kudo Laboratories (Tokyo, Japan) were randomly divided into control $(\mathrm{n}=15)$ and immobilization $(n=30)$ groups. All the rats were housed at $24{ }^{\circ} \mathrm{C}$, with a 12-h light: 12-h dark cycle and with water and food available ad libitum. The rats in the immobilization group were anesthetized with sodium pentobarbital (40 mg/kg), and then the right ankle joint was immobilized in full plantar flexion by plaster casts, whereas the left ankle joint remained free. Periods of joint immobilization were 1,2 , and 4 weeks ( $\mathrm{n}=10$, respectively). Age-matched rats in the control group were observed for equal periods (1, 2, 4 weeks; $n=5)$.

Behavior tests for mechanical sensitivity were performed on the rats before the immobilization (week 0) and at the 1st, 2nd, 3rd and 4th week after the application of immobilization. The test was performed using the home-made restrainer made in cloth because rats in the immobilization group could not walk using hindlimb due to the ankle joint contracture. The restrainer allows the animal to dangle safety, positions the legs so as to be free and under no loading (Fig. 1A). Rats were placed individually in the restrainer after the immobilization removing and allowed to acclimate for $20 \mathrm{~min}$ in a quiet room between 15:00-18:00; room temperature was maintained at $24^{\circ} \mathrm{C}$. The glabrous skin of the hind paw was probed 10 times with a von Frey filament (15 g; North Coast Medical, Morgan Hill, California) at intervals of 10 seconds. Lifting or pulling back of the paw was counted as the paw withdrawal response (PWR) by single experimenter. After the experimental periods, the rats were deeply anesthetized with an overdose of sodium pentobarbital $(40 \mathrm{mg} / \mathrm{kg})$, and transcardial perfusion was performed with saline followed by $4 \%$ paraformaldehyde in a 0.1-M phosphate buffer. Subsequently, plantar glabrous skin tissue of the hind paw $(10 \times 10 \mathrm{~mm})$ was sampled bilaterally. After a soaking in $10 \%$ and $20 \%$ sucrose for $24 \mathrm{~h}$, the tissues were embedded in tragacanth gum, frozen, and stored at $-80{ }^{\circ} \mathrm{C}$. The frozen sections (10 and $30 \mu \mathrm{m}$ ) of skin tissue were cut using a cryostat (CM1950, Leica). Five sections $(10 \mathrm{~mm})$ from each sample (1 $\mathrm{mm}$ apart) were stained with hematoxylineosin $(H \& E)$, and one skin tissue image $(\times 200)$ of the central domain in each footpad was photographed in each section. The epidermal thickness was defined as the distance between the inner and outer borders of the basal and granular layers of the epidermis, respectively. The measurement was conducted at 7 random points on each image using the Image J software (Scion Corp., Frederick, MD). Immunohistochemical staining for the peripheral nerves was performed on the frozen sections $(30 \mu \mathrm{m})$ of skin tissue using the polyclonal anti-PGP 9.5 antibody (NE1013, Calbiochem $\left.{ }^{\circledR}\right)$. Briefly, sections were blocked with $10 \%$ bovine albumin in PBS ( $\mathrm{pH} 7.4$ ) for $30 \mathrm{~min}$ and then incubated overnight with anti-PGP 9.5 
antibodies $(1: 1000)$. After rinsing in PBS, the sections were incubated with biotinylated goat anti-rabbit IgG for $1 \mathrm{~h}$ (1:1000; Vector Lab). Antibody binding was visualized with avidin-biotin-peroxidase complex (VECTASTAIN® Elite kit; Vector Lab) and 3,3'-diaminobenzidinetetrahydrochloride substrate (Sigma). All PGP9.5-positive nerve fiber fragments in the upper dermis $(0-50 \mu \mathrm{m}$ in depth under the epidermis) were counted in the skin tissues images $(\times 200)$ in 5 sections taken at $1 \mathrm{~mm}$ intervals per skin tissue. As a single undulating and branching nerve fiber may present itself more than once, the numbers reported are referred to as nerve fiber profiles (Wallengren and Sundler 2004). The number of nerve fiber profiles per unit area $\left(5000 \mu \mathrm{m}^{2}\right)$ was calculated. All the data are presented as mean \pm S.E. Differences between the control and immobilization groups were assessed using unpaired $t$ tests at each immobilization period. The differences were considered significant at $\mathrm{p}<0.05$.

In the ipsilateral (right) paw of the immobilization group, percent PWR at the 15-g threshold increased steadily after immobilization. By 2 weeks, this increase reached statistical significance and remained significantly different from the control group up to 4 weeks after the immobilization (Fig. 1B). In contrast, meaningful changes were not observed in the contralateral (left) paw in the control or immobilization groups over the entire experimental period (Fig. 1C). Although the experimenter obviously could not be blinded with regard to animals and paws, it was easy to judge the paw withdrawal response (PWR) of rats in the restrainer, and the increase in the percent PWR in the ipsilateral paw of the immobilization group was remarkable. Therefore, the possibility of experimental bias is considered to be minimal. In the change in skin surface, the rough and scaling appearance of the stratum corneum was also confirmed (Fig. 2A). The mean epidermal thickness of the ipsilateral paw of the immobilization group reduced gradually according to the extension of the immobilization period. The significant difference between the control and immobilization groups was observed after 2 and 4 weeks of immobilization (Fig. 2B). The analysis of PGP9.5-positive nerve fibers revealed that the profiles of peripheral nerves in the upper dermis increased gradually in the ipsilateral paw of the immobilization group (Fig. 2D). The significant difference between the immobilization and control groups was observed after 2 and 4 weeks of immobilization. In the contralateral paw, the epidermal thickness and the profiles of the peripheral nerves in the upper dermis showed no change for any of the immobilization periods (Fig. 2C and 2E).

Stankovic et al. (1999) reported that epidermal thinning occurred by crush injury, neurotomy and suture, or neurotomy of the sciatic nerve in rats. They concluded that epidermal thinning is caused by motor deficiency rather than by deficient innervation of the skin (Stankovic et al. 1999). Epidermal thinning was also shown in the experimental rat model with chronic venous insufficiency (Metcalfe et al. 2006). Although this alternative explanation involving circulatory disturbances caused by joint immobilization cannot be excluded, symptoms related to circulatory disturbances, such as edema and gangrene, were not observed in the rats of the immobilization group during the immobilization periods in this study. The time course of epidermal thinning was similar to that of hypersensitivity, with significant differences between the immobilization and control groups after 2 weeks of immobilization, which became even more remarkable at 4 weeks of immobilization. The relationship between epidermal thickness and the mechanical nociceptive threshold is completely unknown.

Here, we also found that the joint immobilization resulted in an increase in the number of peripheral nerves in the upper dermis. Previous studies investigating the effects of capsaicin on skin in healthy subjects showed that capsaicin caused a reduction in the density of peripheral nerves in the skin and that this reduction was associated with a sensory deficit (Nolano et al. 1999, Kennedy et al. 2010, Selim et al. 2010). In contrast, another study suggested that excessive sprouting and an increase in the density of sensory/autonomic nerve fibers induce an increase in painful behaviors in a neuropathic pain rat model (Grelik et al. 2005). According to the theory in those studies, the number of peripheral nerves profiles correlates with nociceptive threshold. The time course of the increase in peripheral nerves was similar to that of hypersensitivity and epidermal thinning with the differences between the immobilization and control groups being recognized at 2 weeks and becoming more remarkable up to 4 weeks after the immobilization.

Epidermal thinning or increase in peripheral nerve profiles in the skin are also observed in other painful disorders; peripheral diabetic neuropathy, atopic dermatitis and Complex regional pain syndrome (Albrecht et al. 2006, Bigliardi-Qi et al. 2007, Petrofsky et al. 2008, Chao et al. 2011). Our results suggest that changes in epidermal thickness and/or peripheral nerves 
in the upper dermis might be partly responsible for immobilization-induced hypersensitivity.

\section{Conflict of Interest}

There is no conflict of interest.

\section{Acknowledgements}

This study was supported by a grant-in-aid for scientific research (No. 23500587) from the Japan Society for the Promotion of Science.

\section{A}

Immobilization
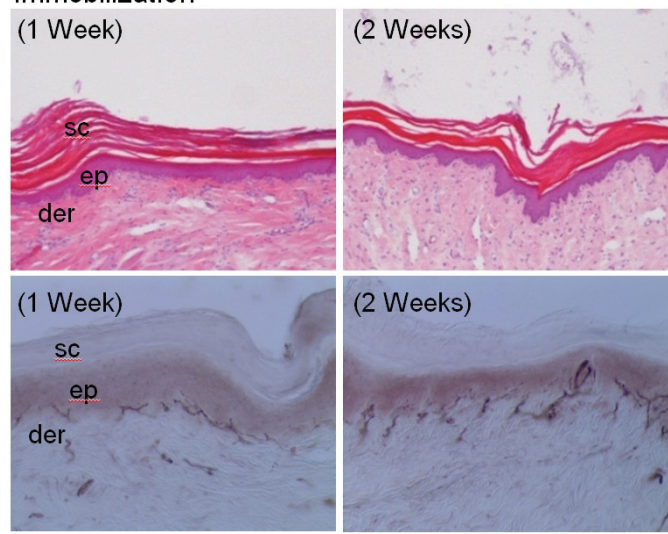

B

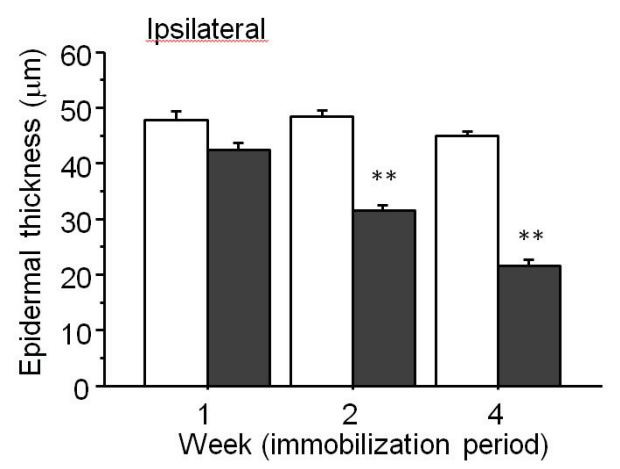

D

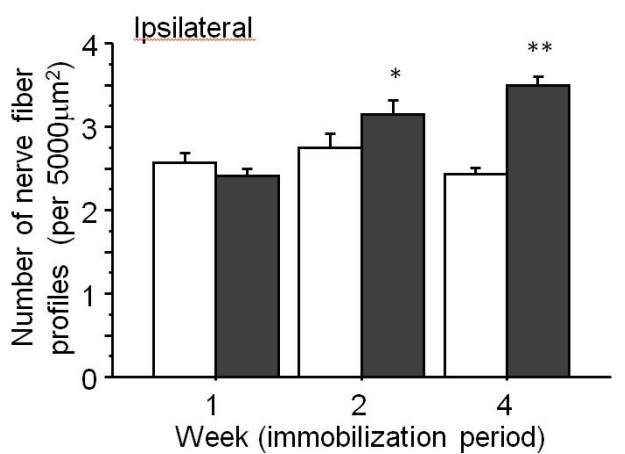

Control
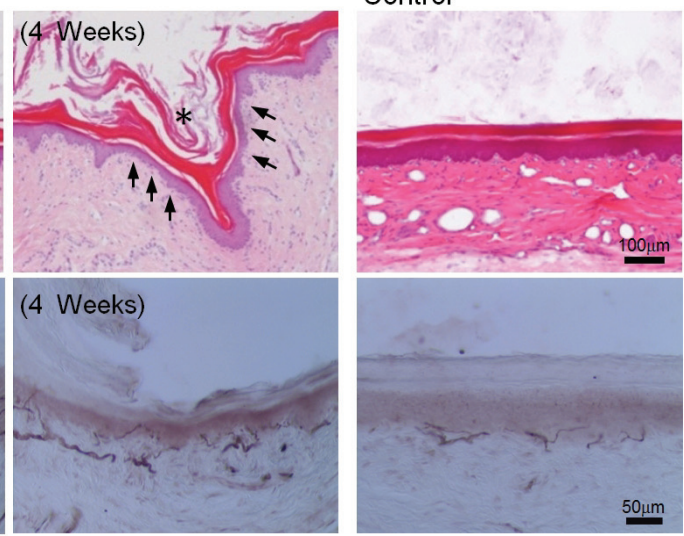

C

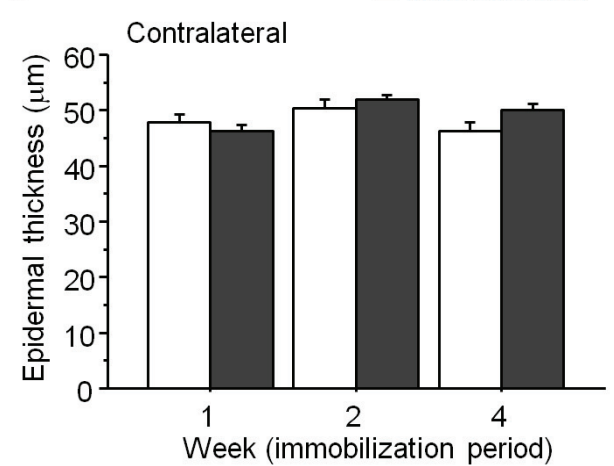

E

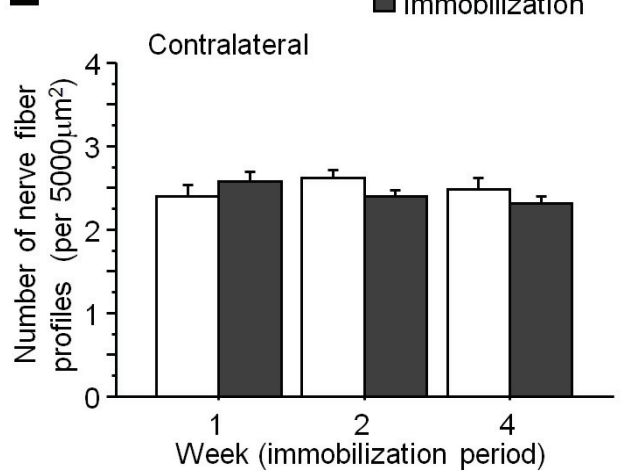

Fig. 2. Skin morphological properties and changes in epidermal thickness of the ipsilateral and contralateral glabrous skin of the hind paw. The photographs of the ipsilateral skin sections with H\&E staining (upper panels) and anti-PGP 9.5 immunohistochemical staining (lower panels) in the immobilization group are shown (A). The photograph of control group at 1 week is shown as a representative example. The scale bars $=100 \mu \mathrm{m}$ in upper panels, $50 \mu \mathrm{m}$ in lower panels. Note a rough and scaling appearance of the stratum corneum (asterisk) and the epidermal thinning (arrows) in the ipsilateral paw, especially after 4 weeks of immobilization. The mean epidermal thickness in the immobilization group reduced gradually according to the length of the immobilization period in the ipsilateral but not in the contralateral hind paw (B and $\mathbf{C}$ ). The numbers of PGP9.5-positive nerve fiber profiles were counted in the upper dermis ( $\mathbf{D}$ and $\mathbf{E}$ ). The number of nerve fiber profiles in the ipsilateral hind paw of the immobilization group was significantly increased compared with that of the control group after 2 and 4 weeks of immobilization. ${ }^{*} \mathrm{p}<0.05,{ }^{*} \mathrm{p}<0.001$. sc, stratum corneum; er, epidermis; der, upper dermis. 


\section{References}

ALBRECHT PJ, HINES S, EISENBERG E, PUD D, FINLAY DR, CONNOLLY MK, PARE M, DAVAR G, RICE FL: Pathologic alterations of cutaneous innervation and vasculature in affected limbs from patients with complex regional pain syndrome. Pain 120: 244-266, 2006.

BIGLIARDI-QI M, GAVERIAUX-RUFF C, PFALTZ K, BADY P, BAUMANN T, RUFLI T, KIEFFER BL, BIGLIARDI PL: Deletion of mu- and kappa-opioid receptors in mice changes epidermal hypertrophy, density of peripheral nerve endings, and itch behavior. J Invest Dermatol 127: 1479-1488, 2007.

CHAO CY, ZHENG YP, CHEING GL: Epidermal thickness and biomechanical properties of plantar tissues in diabetic foot. Ultrasound Med Biol 37: 1029-1038, 2011.

GRELIK C, BENNETT GJ, RIBEIRO-DA-SILVA A: Autonomic fibre sprouting and changes in nociceptive sensory innervation in the rat lower lip skin following chronic constriction injury. Eur J Neurosci 21: 2475-2487, 2005.

GUO TZ, OFFLEY SC, BOYD EA, JACOBS CR, KINGERY WS: Substance P signaling contributes to the vascular and nociceptive abnormalities observed in a tibial fracture rat model of complex regional pain syndrome type I. Pain 108: 95-107, 2004.

KENNEDY WR, VANHOVE GF, LU SP, TOBIAS J, BLEY KR, WALK D, WENDELSCHAFER-CRABB G, SIMONE DA, SELIM MM: A randomized, controlled, open-label study of the long-term effects of NGX-4010, a high-concentration capsaicin patch, on epidermal nerve fiber density and sensory function in healthy volunteers. J Pain 11: 579-587, 2010.

METCALFE MJ, BAKER DM, BURNSTOCK G: Purinoceptor expression on keratinocytes reflects their function on the epidermis during chronic venous insufficiency. Arch Dermatol Res 298: 301-307, 2006.

NOLANO M, SIMONE DA, WENDELSCHAFER-CRABB G, JOHNSON T, HAZEN E, KENNEDY WR: Topical capsaicin in humans: parallel loss of epidermal nerve fibers and pain sensation. Pain 81: 135-145, 1999.

PETROFSKY JS, MCLELLAN K, BAINS GS, PROWSE M, ETHIRAJU G, LEE S, GUNDA S, LOHMAN E, SCHWAB E: Skin heat dissipation: the influence of diabetes, skin thickness, and subcutaneous fat thickness. Diabetes Technol Ther 10: 487-493, 2008.

SELIM MM, WENDELSCHAFER-CRABB G, HODGES JS, SIMONE DA, FOSTER SX, VANHOVE GF, KENNEDY WR: Variation in quantitative sensory testing and epidermal nerve fiber density in repeated measurements. Pain 151: 575-581, 2010.

STANKOVIC N, JOHANSSON O, OQVIST G, HILDEBRAND C: Indirect effect of sciatic nerve injury on the epidermal thickness of plantar glabrous skin in rats. Scand J Plast Reconstr Surg Hand Surg 33: 273-279, 1999.

TERKELSEN AJ, BACH FW, JENSEN TS: Experimental forearm immobilization in humans induces cold and mechanical hyperalgesia. Anesthesiology 109: 297-307, 2008.

USHIDA T, WILLIS WD: Changes in dorsal horn neuronal responses in an experimental wrist contracture model. J Orthop Sci 6: 46-52, 2001.

WALLENGREN J, SUNDLER F: Phototherapy reduces the number of epidermal and CGRP-positive dermal nerve fibres. Acta Derm Venereol 84: 111-115, 2004. 